\title{
Prevention and Control Practices for Human Noroviruses in Long-term Care Facilities in South Carolina
}

\author{
Cortney M. Leone, MS, ${ }^{\mathrm{a}}$ Lalani Jayasekara, BS, ${ }^{\mathrm{a}}$ Julia Sharp, $\mathrm{PhD},{ }^{\mathrm{b}}$ and Angela Fraser, $\mathrm{PhD}{ }^{\mathrm{a}}$
}

Affiliations: a Department of Food, Nutrition, and Packaging Sciences, Clemson University, Clemson, SC; ${ }^{b}$ Department of Mathematical Sciences, Clemson University, Clemson, SC

Address correspondence to: Angela M. Fraser, PhD, Department of Food, Nutrition, and Packaging Sciences, 206 Poole Agriculture Center, Clemson University, Clemson, SC 29634. Email: afraser@clemson.edu

Phone: 864-656-3652 


\section{ABSTRACT}

15 Background: Long-term care facilities (LTCF) are the most common setting for human norovirus (HuNoV) outbreaks. Our study aimed to determine gaps in prevention and control

17 practices for HuNoV in LTCF in South Carolina (SC).

18 Methods: Two researchers visited a convenience sample of 26 LTCF in SC during July-

19 November 2013. Directors were interviewed to determine facility prevention and control

20 practices. Relative frequencies and means were calculated using SAS 9.3.

21 Results: Most Directors had little knowledge of proper sanitizing and disinfecting products and

22 reported missing written procedures for cleaning staff/visitor bathrooms. Only 18 had

23 recommendations for when residents should wash hands. Many used the wrong products for

24 pathogen removal after vomit/fecal events, had no written procedures for cleaning contaminated

25 soft surfaces, did not remove other individuals during clean-up of vomit/fecal episodes, and did

26 not clean a large area surrounding vomit/fecal episodes. Eighteen did not assign specific staff to

27 care for sick; 16 did not designate specific toilets for sick; and 15 did not restrict visitors during

28 an outbreak.

29 Conclusion: Directors' responses indicated gaps in prevention and control practices for HuNoV 30 in LTCF in SC.

32 Key Words: long-term care, human noroviruses, prevention, control, infectious disease 


\section{Prevention and Control Practices for Human Noroviruses in Long-term Care Facilities in South Carolina} gastroenteritis (AGE), sickening 21 million people each year. ${ }^{1}$ Transmission can occur directly person-to-person or indirectly via contaminated surfaces or aerosolized vomitus. Long-term care

42 facilities (LTCF), home to two million Americans, ${ }^{2}$ are the most common setting for HuNoV outbreaks. $^{3}$

Older adults, who represent most residents living in LTCF, are at high risk for AGE, such as $\mathrm{HuNoV}$ infections, because they frequently are immunocompromised or have age-related medical comorbidities. ${ }^{4}$ Moreover, they are at high risk for complications due to HuNoV

47 infections, such as hospitalization and death. ${ }^{5}$ The close living arrangements of older adults in LTCF and their contact with visitors and staff provide many opportunities for both direct (person-to-person) and indirect (environmental) transmission of HuNoV. Our study aimed to determine gaps in prevention and control practices for HuNoV in LTCF in South Carolina (SC).

\section{METHODS}

All methods used in this study were approved by the Clemson University Institutional

53 Review Board (IRB). Informed consent was obtained from all participants before data collection 54 began.

\section{Site Selection}

A list of all registered LTCF (N=197) was obtained from the SC Department of Health

57 and Environmental Control website in June 2013. An Internet search was performed to determine 58 whether each facility met our eligibility criteria: facilities must offer skilled nursing care, be 
59 licensed by the state of SC, operate year round, primarily serve older adults $\geq 60$ years, be a

60 residential facility, not provide care only for a specific population such as Alzheimer's patients,

61 and prepare and serve meals in a cafeteria or to individual rooms. After the initial Internet search,

6234 LTCF were excluded.

The 163 eligible facilities were called up to 4 times and asked to participate. Thirty-nine (39) were not interested, 11 stated their corporate offices did not allow participation in research studies, and 78 never responded. Eight stated they were interested but could not schedule a site visit for various reasons. A total of 27 facility visits were performed. One facility only served

67 mentally ill patients, so our final sample size was $26 \mathrm{LTCF}$, which is a participation rate of $16 \%$ $68(26 / 163)$.

\section{Interviews}

Interviews with facility directors and/or their designees (e.g., infection control nurse,

71 director of nursing, or head of housekeeping) were conducted between July and November 2013.

72 During a 60-minute interview, directors/designees (hereafter called Directors) answered 44

73 questions that focused on infection prevention and control practices. Specifically, we asked

74 questions related to prevention (general hygiene and sanitation), control (handling of vomit and

75 fecal matter), and infectious disease control during an outbreak. All questions were based on the

76 U.S. Centers for Disease Control and Prevention (CDC) recommended best practices for

77 preventing norovirus infections in healthcare facilities. ${ }^{6}$ Responses were hand-recorded as "yes"

78 or "no" and comments noted. If a Director reported their facility had a written policy or

79 procedure, they were asked to show it to the interviewer. The Director also independently

80 completed a questionnaire about facility characteristics, worker training, and personal

81 demographics. 


\section{Data Management and Analysis}

All interview and questionnaire responses were coded and entered into a Microsoft Excel spreadsheet as numeric values. Handwritten comments captured during the interview were categorized into themes, which were then converted to numeric values. Relative frequencies were calculated for categorical variables, and means were calculated for continuous variables using SAS 9.3 for Windows (SAS Institute Inc., Cary, NC). Proportions of responses between for-profit and non-profit facilities were compared using Fisher's Exact Test because the sample size was small. A significance level of 0.05 was used for all tests of significance.

\section{RESULTS}

Our results include a description of facility and Director characteristics. We also present the frequency of responses about practices for all facilities as well as for non-profit and for-profit facilities to determine if practices were associated with facility type.

\section{Facility and Director Characteristics}

Sixteen Directors indicated their facility was a skilled nursing facility, 9 a continuing care community, 8 a nursing home, and 3 an assisted living facility. This number is greater than 26 because respondents could select more than one choice for this questionnaire item. Most (19) facilities were for-profit (corporate or independently owned); 6 were non-profit (government or faith-based) institutions; and 1 did not select a facility type. Directors reported an average of 117 staff (range 44-225) (e.g., health care, food service, and custodial), 89 residents (range 16-254), and 102 beds (range 30-282). All provided training on infectious disease control.

In 5 facilities, the facility director was interviewed and in 15 the interview was conducted with the director of nursing or the infection control nurse. In 6 , both the director and the infection 
104 control nurse were interviewed. Sixteen Directors had 1-5 years of experience and 10 had

105 completed an associate's degree or bachelor's degree.

106 Practices: General Hygiene and Sanitation

107 Our study findings indicated 3 gaps in general cleaning and sanitation practices: 1)

108 improper use of sanitizing or disinfecting products after contamination events, 2) no written

109 procedure for cleaning staff/visitor bathrooms to prevent transmission of $\mathrm{HuNoV}$, and 3) no list

110 of contamination events after which residents should wash hands. All Directors reported their

111 facility followed a general schedule for cleaning and 18 reported some type of deep cleaning.

112 Half (13) reported their facility was cleaned during the first shift only, and an additional 13

113 cleaned during the second or third shifts. For hard, food-contact surfaces, 13 reported using

114 quaternary ammonium-based sanitizers, 5 chlorine-based sanitizers, and 4 sanitizers with other

115 active ingredients (data not reported). Four Directors stated "I don't know" or "unsure" when

116 asked about the type of sanitizer used on hard, food-contact surfaces. For other hard surfaces, 11

117 reported using quaternary ammonium-based sanitizers or disinfectants, 10 chlorine-based, and 3

118 sanitizers or disinfectants with other active ingredients. One reported using both chlorine-based

119 and quaternary ammonium-based sanitizers and disinfectants for other hard, non-food-contact

120 surfaces and 1 did not know. Twenty-two (22) facilities reported having written procedures for

121 cleaning resident bathrooms, including bedside commodes, but only 1 had a written procedure

122 for cleaning staff/visitor bathrooms (Table 1). 
Table 1: General Hygiene and Sanitation at Long-term Care Facilities in South Carolina

\begin{tabular}{|c|c|c|c|c|c|c|c|c|c|c|c|c|c|c|c|c|c|c|}
\hline \multirow[b]{3}{*}{ Characteristics } & \multicolumn{6}{|c|}{ For-profit $(\mathbf{n}=19)^{*}$} & \multicolumn{6}{|c|}{ Non-profit $(\mathrm{n}=6)^{*}$} & \multicolumn{6}{|c|}{ All facilities $(\mathrm{N}=26)$} \\
\hline & \multicolumn{2}{|c|}{ Yes } & \multicolumn{2}{|c|}{ No } & \multicolumn{2}{|c|}{ Other } & \multicolumn{2}{|c|}{ Yes } & \multicolumn{2}{|c|}{ No } & \multicolumn{2}{|c|}{ Other } & \multicolumn{2}{|c|}{ Yes } & \multicolumn{2}{|c|}{ No } & \multicolumn{2}{|c|}{ Other } \\
\hline & $\mathbf{n}$ & $\%$ & $\mathbf{n}$ & $\%$ & $\mathbf{n}$ & $\%$ & $\mathbf{n}$ & $\%$ & $\mathbf{n}$ & $\%$ & $\mathbf{n}$ & $\%$ & $\mathbf{n}$ & $\%$ & $\mathbf{n}$ & $\%$ & $\mathbf{n}$ & $\%$ \\
\hline \multicolumn{19}{|l|}{ Bathroom Cleaning and Laundry } \\
\hline \multicolumn{19}{|l|}{ Written procedures for: } \\
\hline Resident bathrooms & 15 & 79.0 & 3 & 15.8 & 1 & 5.3 & 6 & 0.0 & 0 & 0.0 & 0 & 0.0 & 22 & 84.6 & 3 & 11.5 & 1 & 3.9 \\
\hline Staff/visitor bathrooms & 0 & 0.0 & 17 & 89.5 & 2 & 10.5 & 1 & 16.7 & 5 & 83.3 & 0 & 0.0 & 1 & 3.9 & 23 & 88.5 & 2 & 7.7 \\
\hline Washing laundry & 17 & 89.5 & 1 & 5.3 & 1 & 5.3 & 6 & 100.0 & 0 & 0.0 & 0 & 0.0 & 24 & 92.3 & 1 & 3.9 & 1 & 3.9 \\
\hline \multicolumn{19}{|l|}{ Laundry practices } \\
\hline $\begin{array}{l}\text { Laundry rooms separated from other } \\
\text { areas }\end{array}$ & 19 & 100.0 & 0 & 0.0 & 0 & 0.0 & 6 & 100.0 & 0 & 0.0 & 0 & 0.0 & 26 & 100.0 & 0 & 0.0 & 0 & 0.0 \\
\hline $\begin{array}{l}\text { Soiled and non-soiled laundry } \\
\text { transported separately }\end{array}$ & 19 & 100.0 & 0 & 0.0 & 0 & 0.0 & 6 & 100.0 & 0 & 0.0 & 0 & 0.0 & 26 & 100.0 & 0 & 0.0 & 0 & 0.0 \\
\hline $\begin{array}{l}\text { Bleach/other sanitizing agents added } \\
\text { to wash cycle }\end{array}$ & 17 & 89.5 & 0 & 0.0 & 2 & 10.5 & 6 & 100.0 & 0 & 0.0 & 0 & 0.0 & 24 & 92.3 & 0 & 0.0 & 2 & 7.7 \\
\hline \multicolumn{19}{|l|}{ Personal Hygiene } \\
\hline \multicolumn{19}{|l|}{ Written procedures for: } \\
\hline Dress code & 19 & 100.0 & 0 & 0.0 & 0 & 0.0 & 6 & 100.0 & 0 & 0.0 & 0 & 0.0 & 26 & 100.0 & 0 & 0.0 & 0 & 0.0 \\
\hline Fingernail grooming & 17 & 89.5 & 1 & 5.3 & 1 & 5.3 & 6 & 100.0 & 0 & 0.0 & 0 & 0.0 & 24 & 92.3 & 1 & 3.9 & 1 & 3.9 \\
\hline Jewelry† & 16 & 84.2 & 2 & 10.5 & 0 & 0.0 & 6 & 100.0 & 0 & 0.0 & 0 & 0.0 & 23 & 88.5 & 2 & 7.7 & 0 & 0.0 \\
\hline \multicolumn{19}{|l|}{ Hand hygiene practices } \\
\hline $\begin{array}{l}\text { Employees wash hands/use hand } \\
\text { sanitizer after certain activities }\end{array}$ & 19 & 100.0 & 0 & 0.0 & 0 & 0.0 & 6 & 100.0 & 0 & 0.0 & 0 & 0.0 & 26 & 100.0 & 0 & 0.0 & 0 & 0.0 \\
\hline $\begin{array}{l}\text { Employees use hand sanitizer when } \\
\text { hand hygiene is not required }\end{array}$ & 19 & 100.0 & 0 & 0.0 & 0 & 0.0 & 6 & 100.0 & 0 & 0.0 & 0 & 0.0 & 26 & 100.0 & 0 & 0.0 & 0 & 0.0 \\
\hline $\begin{array}{l}\text { Residents wash hands after certain } \\
\text { activities }\end{array}$ & 13 & 68.4 & 5 & 26.3 & 1 & 5.3 & 4 & 66.7 & 2 & 33.3 & 0 & 0.0 & 18 & 69.2 & 7 & 26.9 & 1 & 3.9 \\
\hline
\end{tabular}

124 *One facility did not indicate the business type.

125 †Some directors did not provide an answer. 
All Directors reported laundry was washed on site with 9 washing resident clothing only

127 and 16 washing linens and mop heads in addition to resident clothing. Across all facilities,

128 laundry rooms were separate from resident rooms, kitchens, and serving and commons areas.

129 When transporting laundry throughout the facility, all reported using covered carts to separate

130 clean and dirty laundry as well as contaminated and non-contaminated laundry. Nearly all (24)

131 reported having written procedures for how to wash laundry. Of those 24 , all required adding a

132 sanitizing agent to the wash cycle, but only 15 specified a sanitizer, such as chlorine bleach.

All had a written dress code and allowed employees to arrive to work in their uniform.

134 Nearly all (23) had recommendations on wearing jewelry and fingernail grooming. Hand hygiene

135 of workers after certain activities was required by all, with 18 identifying activities after which

136 residents needed to wash their hands.

137 Practices: Handling of Vomit/Fecal Matter

Gaps (5) were also found in practices related to handling vomit/fecal matter. These

139 included: 1) no written procedures for cleaning bathrooms after a vomit/fecal episode, 2) use of

140 ineffective products for pathogen removal after a vomit/fecal episode, 3) no written procedures

141 for cleaning contaminated soft surfaces, 4) not removing other individuals during clean-up of a

142 vomit/fecal episode, and 5) not cleaning a large area surrounding a vomit/fecal episode.

143 Nearly all (25) reported vomit and diarrheal episodes occurred outside a bathroom. Most

144 stated that episodes of vomit (20) and diarrhea (24) occur most often in resident rooms. Twenty-

145 four (24) reported they had a facility policy on incontinence care, but only 19 had procedures for

146 clean-up of vomit or fecal matter on environmental surfaces (Table 2). Eighteen had written

147 procedures for cleaning linens contaminated with vomit or feces as well as written procedures for

148 cleaning hard, non-porous surfaces after exposure to vomit or fecal matter. Nearly half (12) had 
Table 2: Handling of Vomit/Fecal Matter at Long-term Care Facilities in South Carolina

\begin{tabular}{|c|c|c|c|c|c|c|c|c|c|c|c|c|c|c|c|c|c|c|}
\hline \multirow[b]{3}{*}{ Characteristics } & \multicolumn{6}{|c|}{ For-profit (n=19)* } & \multicolumn{6}{|c|}{ Non-profit $(\mathrm{n}=6)^{*}$} & \multicolumn{6}{|c|}{ All facilities $(\mathrm{N}=26)$} \\
\hline & \multicolumn{2}{|c|}{ Yes } & \multicolumn{2}{|c|}{ No } & \multicolumn{2}{|c|}{ Other } & \multicolumn{2}{|c|}{ Yes } & \multicolumn{2}{|c|}{ No } & \multicolumn{2}{|c|}{ Other } & \multicolumn{2}{|c|}{ Yes } & \multicolumn{2}{|c|}{ No } & \multicolumn{2}{|c|}{ Other } \\
\hline & $\mathbf{n}$ & $\%$ & $\mathbf{n}$ & $\%$ & $\mathbf{n}$ & $\%$ & $\mathbf{n}$ & $\%$ & $\mathbf{n}$ & $\%$ & $\mathbf{n}$ & $\%$ & $\mathbf{n}$ & $\%$ & $\mathbf{n}$ & $\%$ & $\mathbf{n}$ & $\%$ \\
\hline Written procedures for cleaning: & & & & & & & & & & & & & & & & & & \\
\hline Soiled resident & 17 & 89.5 & 2 & 10.5 & 0 & 0.0 & 6 & 100.0 & 0 & 0.0 & 0 & 0.0 & 24 & 92.3 & 2 & 7.7 & 0 & 0.0 \\
\hline Vomit/fecal matter & 13 & 68.4 & 3 & 15.8 & 3 & 15.8 & 5 & 83.3 & 1 & 16.7 & 0 & 0.0 & 19 & 73.1 & 4 & 15.4 & 3 & 11.5 \\
\hline Contaminated linens/clothing & 14 & 73.7 & 2 & 10.5 & 3 & 15.8 & 4 & 66.7 & 1 & 16.7 & 1 & 16.7 & 18 & 69.2 & 4 & 15.4 & 4 & 15.4 \\
\hline Contaminated hard surfaces & 13 & 68.4 & 4 & 21.1 & 2 & 10.5 & 4 & 66.7 & 2 & 33.3 & 0 & 0.0 & 18 & 69.2 & 6 & 23.1 & 2 & 7.7 \\
\hline $\begin{array}{l}\text { Contaminated upholstered furniture, } \\
\text { carpets, and rugs }\end{array}$ & 7 & 36.8 & 11 & 57.9 & 1 & 5.3 & 4 & 66.7 & 2 & 33.3 & 0 & 0.0 & 12 & 46.2 & 13 & 50.0 & 1 & 3.9 \\
\hline Clean-up practices & & & & & & & & & & & & & & & & & & \\
\hline $\begin{array}{l}\text { Other individuals removed from room } \\
\text { during clean-up }\end{array}$ & 9 & 47.4 & 8 & 42.1 & 2 & 10.5 & 2 & 33.3 & 3 & 50.0 & 1 & 16.7 & 11 & 42.3 & 12 & 46.2 & 3 & 11.5 \\
\hline $\begin{array}{l}\text { Workers and/or residents changed } \\
\text { clothes after an episode }\end{array}$ & 15 & 79.0 & 3 & 15.8 & 1 & 5.3 & 3 & 50.0 & 2 & 33.3 & 1 & 16.7 & 19 & 73.1 & 5 & 19.2 & 2 & 7.7 \\
\hline $\begin{array}{l}\text { Written procedures on: } \\
\text { Cleaning restrooms after } \\
\text { vomiting/diarrheal episodes }\end{array}$ & 6 & 31.6 & 11 & 57.9 & 2 & 10.5 & 2 & 33.3 & 4 & 66.7 & 0 & 0.0 & 9 & 34.6 & 15 & 57.7 & 2 & 7.7 \\
\hline
\end{tabular}


151 written procedures for cleaning upholstered furniture, carpets, and rugs after exposure to vomit

152 or fecal matter, and 9 had different procedures for cleaning bathrooms after vomiting or diarrheal

153 episodes. Less than half (11) reported that other individuals were removed from the room during

154 clean-up of feces and vomit. Eight facilities stipulated a wide area surrounding an episode of

155 vomit/fecal matter be cleaned while 18 cleaned only the vomit/fecal matter episode. Exact

156 dimensions were not provided by any.

157 Practices: Infectious Disease Control

Most (22) reported they disallowed sick residents in common areas, and 20 disallowed

159 healthy residents to visit sick residents (Table 3). Only 9 designated specific toilets for sick

160 residents. Nearly all (25) excluded sick staff from work, and 8 assigned specific staff members to

161 care for sick residents. Visitors were not allowed to have contact with sick residents in 11

162 facilities.

163 All Directors stated employees were required to wear disposable gloves when caring for

164 sick residents. Nearly all (24) required employees to wear plastic aprons or cloth gowns, and 19

165 required wearing masks when caring for sick residents (Table 3). Only 9 reported using shoe

166 covers when entering the room of sick residents. Employees at 25 facilities were required to

167 remove personal protective equipment before leaving a room and dispose of these articles in

168 biohazard (13) or other specified containers (13).

169 Our results showed no significant differences between reported practices at for-profit

170 versus non-profit facilities. MUST BE TWO SENTENCES. 
Table 3: Infectious Disease Control at Long-term Care Facilities in South Carolina

\begin{tabular}{|c|c|c|c|c|c|c|c|c|c|c|c|c|c|c|c|c|c|c|}
\hline \multirow[b]{3}{*}{ Characteristics } & \multicolumn{6}{|c|}{ For-profit $(\mathbf{n}=19) *$} & \multicolumn{6}{|c|}{ Non-profit $(\mathrm{n}=6)^{*}$} & \multicolumn{6}{|c|}{ All facilities (N=26) } \\
\hline & \multicolumn{2}{|c|}{ Yes } & \multicolumn{2}{|c|}{ No } & \multicolumn{2}{|c|}{ Other } & \multicolumn{2}{|c|}{ Yes } & \multicolumn{2}{|c|}{ No } & \multicolumn{2}{|c|}{ Other } & \multicolumn{2}{|c|}{ Yes } & \multicolumn{2}{|c|}{ No } & \multicolumn{2}{|c|}{ Other } \\
\hline & $\mathbf{n}$ & $\%$ & $\mathbf{n}$ & $\%$ & $\mathbf{n}$ & $\%$ & $\mathbf{n}$ & $\%$ & $\mathbf{n}$ & $\%$ & $\mathbf{n}$ & $\%$ & $\mathbf{n}$ & $\%$ & $\mathbf{n}$ & $\%$ & $\mathbf{n}$ & $\%$ \\
\hline Practices for handling sick residents & & & & & & & & & & & & & & & & & & \\
\hline Not allowed in the commons areas & 16 & 84.2 & 1 & 5.3 & 2 & 10.5 & 5 & 83.3 & 1 & 16.7 & 0 & 0.0 & 22 & 84.6 & 2 & 7.7 & 2 & 7.7 \\
\hline Isolated from healthy residents & 16 & 84.2 & 2 & 10.5 & 1 & 5.3 & 5 & 83.3 & 0 & 0.0 & 1 & 16.7 & 22 & 84.6 & 2 & 7.7 & 2 & 7.7 \\
\hline $\begin{array}{l}\text { Not allowed visits from healthy } \\
\text { residents }\end{array}$ & 14 & 73.7 & 3 & 15.8 & 2 & 10.5 & 5 & 83.3 & 1 & 16.7 & 0 & 0.0 & 20 & 76.9 & 4 & 15.4 & 2 & 7.7 \\
\hline Designated to specific toilets & 6 & 31.6 & 12 & 63.2 & 1 & 5.3 & 3 & 50.0 & 3 & 50.0 & 0 & 0.0 & 9 & 34.6 & 16 & 61.5 & 1 & 3.9 \\
\hline Practices for handling staff & & & & & & & & & & & & & & & & & & \\
\hline Sick staff excluded from work & 18 & 94.7 & 0 & 0.0 & 1 & 5.3 & 6 & 100.0 & 0 & 0.0 & 0 & 0.0 & 25 & 96.2 & 0 & 0.0 & 1 & 3.9 \\
\hline $\begin{array}{l}\text { Assign specific staff to only care for } \\
\text { sick residents }\end{array}$ & 6 & 31.6 & 13 & 68.4 & 0 & 0.0 & 1 & 16.7 & 5 & 83.3 & 0 & 0.0 & 8 & 30.8 & 18 & 69.2 & 0 & 0.0 \\
\hline Practices for handling visitors & & & & & & & & & & & & & & & & & & \\
\hline $\begin{array}{l}\text { Not allowed to have contact with } \\
\text { sick residents }\end{array}$ & 9 & 47.4 & 10 & 52.6 & 0 & 0.0 & 2 & 33.3 & 4 & 66.7 & 0 & 0.0 & 11 & 42.3 & 15 & 57.7 & 0 & 0.0 \\
\hline Written procedures on: & & & & & & & & & & & & & & & & & & \\
\hline $\begin{array}{l}\text { Cleaning and disinfecting during an } \\
\text { outbreak }\end{array}$ & 15 & 79.0 & 4 & 21.1 & 0 & 0.0 & 5 & 83.3 & 1 & 16.7 & 0 & 0.0 & 21 & 80.8 & 5 & 19.2 & 0 & 0.0 \\
\hline $\begin{array}{l}\text { Practices for wearing } \mathrm{PPE}^{\ddagger} \text { when caring } \\
\text { for sick residents }\end{array}$ & & & & & & & & & & & & & & & & & & \\
\hline Disposable gloves & 19 & 100.0 & 0 & 0.0 & 0 & 0.0 & 6 & 100.0 & 0 & 0.0 & 0 & 0.0 & 26 & 100.0 & 0 & 0.0 & 0 & 0.0 \\
\hline Apron or gown & 18 & 94.7 & 0 & 0.0 & 1 & 5.3 & 5 & 83.3 & 1 & 16.7 & 0 & 0.0 & 24 & 92.3 & 1 & 3.9 & 1 & 3.9 \\
\hline Masks & 14 & 73.7 & 3 & 15.8 & 2 & 10.5 & 4 & 66.7 & 1 & 16.7 & 1 & 16.7 & 19 & 73.1 & 4 & 15.4 & 3 & 11.5 \\
\hline Shoe covers & 8 & 42.1 & 8 & 42.1 & 3 & 15.8 & 0 & 0.0 & 6 & 100.0 & 0 & 0.0 & 9 & 34.6 & 14 & 53.9 & 3 & 11.5 \\
\hline
\end{tabular}

172 *One facility did not indicate the business type 


\section{DISCUSSION}

Our study aimed to determine prevention and control practices for HuNoV in LTCF in South Carolina (SC) as the evidence on how well LTCF prevent and control HuNoV is limited. Preventing and controlling HuNoV in LTCF is critical because, not only are older adults at a high risk of being infected with $\mathrm{HuNoV}$, they are also at high risk for complications due to HuNoV infections.

\section{Prevention Practices: General Cleaning and Sanitation}

No Directors reported using the correct product for pathogen removal (sanitizers or disinfectants) after specific contamination events. Sanitizers reduce, not eliminate, bacteria whereas disinfectants eliminate a wider range of microorganisms including fungi, viruses, and bacteria but not necessarily spores. ${ }^{7}$ Sanitizers are primarily used on food-contact surfaces and soft surfaces whereas disinfectants are used on non-food-contact surfaces and any surface contaminated with vomit or fecal matter. ${ }^{1,8}$ Therefore, sanitizers or disinfectants can be used every day to treat normal contamination events, but disinfectants should always be used if a surface, regardless of its type, is contaminated with vomit and fecal matter. ${ }^{1}$

HuNoV can be introduced into health-care settings by ill staff/visitors when they use the bathroom while sick. ${ }^{1}$ Only 1 facility had procedures for cleaning staff/visitor bathrooms. Flushing the toilet aerosolizes virus particles allowing them to be deposited onto bathroom surfaces. ${ }^{9}$ Also, when sick individuals do not wash their hands after using the bathroom, they can contaminate surfaces (e.g., door handles, faucet heads, and flush handles). ${ }^{10}$ Healthy persons who contact these contaminated bathroom surfaces can become infected. Moreover, because AGE is mostly caused by non-enveloped viruses, ${ }^{11}$ which are highly resistant to disinfection, ${ }^{12}$ it is important that all bathroom surfaces be cleaned then disinfected with high-level disinfectants as 
197 they eliminate all microorganisms except bacterial spores. Low-level disinfectants eliminate 198 most vegetative bacteria, some fungi, and some viruses. ${ }^{13}$

As hands can be a source of $\mathrm{HuNoV}$, proper handwashing is critical for all persons - staff

200

201

202

203

204

205

206

207

208

209

210

211

212

213

214

215

216

217

218

219 as well as residents. Many LTCF residents are bed-bound, so providing recommendations for them might not be practical, but providing recommendations for all other residents is necessary in preventing $\mathrm{HuNoV}$ transmission in a long-term care setting.

\section{Practices: Handling of Vomit and Fecal Matter}

To eliminate HuNoV, the CDC recommends using sodium hypochlorite (chlorine bleach) at a concentration of $1000-5000 \mathrm{ppm}^{1}$ or a disinfectant registered as effective against $\mathrm{HuNoV}$ by the U.S. Environmental Protection Agency. ${ }^{14}$ Use of products not effective against HuNoV could result in a failure to eliminate $\mathrm{HuNoV}$ on surfaces after a vomit/fecal episode which could cause additional cases of illness or could prolong an existing outbreak.

Less than half of the Directors (10) used chlorine-based products for pathogen removal from hard surfaces with 11 using quaternary ammonium-based products and 3 products with other active ingredients. While quaternary ammonium-based products can be used for general cleaning and sanitizing, they are not effective against $\mathrm{HuNoV}^{10}$ so should not be used to disinfect after vomit/fecal episodes. To eliminate $\mathrm{HuNoV}$, the $\mathrm{CDC}$ recommends using sodium hypochlorite (chlorine bleach) at a concentration of 1000-5000 ppm on hard surfaces. ${ }^{1} \mathrm{We}$ believe one reason many facilities did not use chlorine bleach is that chlorine-based products are not allowed by the SC Occupational Safety and Health Administration (OSHA) because they are classified as corrosive agents. If a facility uses chlorine-based products, they must properly install an eye wash station. ${ }^{15}$ We did not check if facilities in our sample had eye wash stations but presumed most did not. We also believe facilities used quaternary ammonium-based products 
220 because they believe they are not corrosive when in fact they are corrosive. ${ }^{16}$ So, if quaternary 221 ammonium products are used, an eye wash station must be installed per OSHA regulations. Written procedures for treating non-launderable soft surfaces, such as carpets, rugs, and 223 upholstered furniture, is important because these types of surfaces can harbor microorganisms, 224 such as HuNoV, and potentially transmit them to healthy individuals. When HuNoV attach to 225 soft surfaces, they are difficult to eliminate, ${ }^{17}$ and chlorine bleach, which is recommended to 226 eliminate HuNoV from hard surfaces, can damage or discolor the material of soft surfaces.

227 Vomit/fecal matter should be removed from upholstery or carpet immediately following an 228 episode. The CDC recommends using a manufacturer-approved cleaning agent followed by 229 steam cleaning to accomplish this. ${ }^{6}$ However, the efficacy of this practice at eliminating HuNoV 230 has not been proven. It is not practical to eliminate all soft surfaces as older adults need a 231 comfortable and home-like environment. One solution is to use easy-to-clean vinyl upholstered 232 furniture or furniture with removable cushions, which are easier to clean and disinfect. Individuals must also be removed from a room during clean-up of a vomit or diarrheal 234 episode to minimize risk of infection due to exposure. ${ }^{18} \mathrm{HuNoV}$ particles can become 235 aerosolized as a result of the cleaning process. When aerosolized, they can be ingested by 236 individuals in the surrounding area, ${ }^{1}$ so only cleaning staff wearing proper protective equipment 237 (gowns, gloves, face masks, and shoe covers) should be in contaminated rooms, all other 238 individuals must be removed. ${ }^{6}$

The floor can become contaminated via splatter and droplets, ${ }^{19}$ and workers shoes could 240 transmit virus particles from that room to other areas. Wearing shoe covers and removing them 241 before leaving the room could prevent introduction of viruses to other areas. Splatter and 242 droplets from projectile vomiting can spread over a large area and contaminate surfaces that look 
243 clean to the naked eye. ${ }^{19}$ At this time, there is insufficient evidence on how large of an area can 244 become contaminated. However, it is best to be cautious and clean as wide of an area as possible 245 surrounding the vomit/fecal matter.

\section{Practices: Infectious Disease Control}

248 for healthy residents to contact contaminated surfaces as virus particles can become aerosolized 249 after flushing then settle on bathroom surfaces becoming a source of HuNoV. ${ }^{9}$ In addition, 250 specific staff should be assigned to work with sick residents. ${ }^{6}$ One study reported staff spread $251 \mathrm{HuNoV}$ from residents on the second floor, where an outbreak began, to residents on the first

252 floor. ${ }^{20}$ Assigning specific staff to care only for sick residents decreases the likelihood that staff

253 hands and clothing become contaminated and subsequently infect healthy residents or 254 contaminate fomites in healthy resident's rooms.

Non-essential visitors must also be restricted during an outbreak ${ }^{6}$ which can be difficult to 256 implement as Directors in our sample reported that residents want their relatives to visit them 257 when they are sick. Additionally, the CDC recommends screening and excluding visitors with 258 symptoms consistent with a HuNoV infection ${ }^{6}$ to prevent additional introduction of viruses from 259 the community to long-term care residents.

260 Limitations

We used a convenience sample of LTCF in SC so our findings cannot be generalized to

262 all LTCF in SC. The length of site visits (2-3 hours) could have been a limitation. Additionally, 263 some Directors might not have wanted to interrupt essential facility activities more than 264 necessary, so they might not have taken the time to find out answers to questions they were 265 unsure of or interrupted staff that might have been better able to answer such questions. Self- 
266 reported practices are not always accurate. ${ }^{21}$ Because the intent of the interviews was to have

267 Directors self-report facility practices, better practices for prevention and control may have been

268 reported than what actually occurs in facilities.

\section{CONCLUSIONS}

270 LTCF cause nearly $60 \%$ of HuNoV outbreaks in the U.S. so identifying gaps in

271 prevention and control practices is essential to preventing future cases of illness. In our sample of

272 LTCF in SC, specific gaps included lack of self-reported knowledge about sanitizing and

273 disinfecting products, insufficient recommendations for resident hand washing, insufficient

274 procedures for cleaning up after a vomit/fecal episode, and insufficient infection control

275 practices during an outbreak.

276 Outbreaks of HuNoV will continue to occur in LTCF if these gaps in prevention and

277 control practices are not addressed. One way to ensure best practices are being followed is to

278 have accurate and up-to-date policies and procedures in facilities. However, the presence of

279 policies and procedures alone is not sufficient to improve practices. Providing evidence-based,

280 practical education or training for practitioners and other personnel in LTCF is also critical in

281 preventing future outbreaks. Training should emphasize proper use of sanitizing and disinfecting

282 products, procedures for cleaning up vomit/fecal matter, and proper infection control practices

283 during outbreaks of enteric illness. 


\section{ACKNOWLEDGEMENTS}

285 This material is based upon work supported by the Cooperative State Research, Education and

286 Extension Service, U.S. Department of Agriculture, under agreement no. 2009-51110-05858.

287 Any opinions, findings, conclusions, or recommendations expressed in this publication are those

288 of the author(s) and do not necessarily reflect the view of the USDA. 


\section{REFERENCES}

1. Hall AJ, Vinjé J, Lopman B, et al. Updated norovirus outbreak management and disease prevention guidelines. MMWR Morb Mortal Wkly Rep. 2011;60:1-15.

2. Harris-Kojetin L, Sengupta M, Park-Lee E, Valverde R. Long-term care services in the United States: 2013 overview. Hyattsville, MD: National Center for Health Statistics. 2013.

3. Hall AJ, Wikswo ME, Pringle K, Gould LH, Parashar UD. Vital signs: Foodborne norovirus outbreaks - United States, 2009-2012. MMWR Morb Mortal Wkly Rep. 2014;63:491-495.

4. Kirk MD, Veitch MG, Hall GV. Gastroenteritis and food-borne disease in elderly people living in long-term care. Clin Infect Dis. 2010;50:397-404.

5. Trivedi TK, DeSalvo T, Lee L, et al. Hospitalizations and mortality associated with norovirus outbreaks in nursing homes 2009-2010. JAMA. 2012;308:1668-1675.

6. MacCannell T, Umscheid CA, Agarwal RK, et al. Guideline for the prevention and control of norovirus gastroenteritis outbreaks in healthcare settings. Infect Control Hosp Epidemiol. 2011;32:939-969.

7. US Environmental Protection Agency. OCSPP 810.2000: General Considerations for Uses of Antimicrobial Agents. Washington DC: US Environmental Protection Agency; 2012.

8. US Environmental Protection Agency. Antimicrobial Pesticide Products. http://www.epa.gov/opp00001/factsheets/antimic.htm. Accessed June 22, 2015.

9. Barker J, Jones MV. The potential spread of infection caused by aerosol contamination of surfaces after flushing a domestic toilet. J Appl Microbiol. 2005;99:339-347.

10. Barker J, Vipond I, Bloomfield S. Effects of cleaning and disinfection in reducing the spread of norovirus contamination via environmental surfaces. J Hosp Infect. 2004;58:4249.

11. Hall AJ, Lopman BA, Payne DC, Patel MM, Gastañaduy PA, Vinjé J, Parashar UD. Norovirus disease in the United States. Emerg Infect Dis. 2013;19(8).

12. Sattar SA. Hierarchy of susceptibility of viruses to environmental surface disinfectants: a predictor of activity against new and emerging viral pathogens. $J A O A C$ Int. 2007;90(6):1655-1658.

13. Rutala WA, Weber DJ, the Healthcare Infection Control Practices Advisory Committee (HICPAC). Guideline for Disinfection and Sterilization in Healthcare Facilities, 2008. Atlanta, GA: The Centers for Disease Control and Prevention; 2009.

14. US Environmental Protection Agency. List G: EPA Registered Hospital Disinfectants Effective Against Norovirus (Norwalk-like virus). http://www.epa.gov/oppad001/ list_g_norovirus.pdf. Accessed June 22, 2015.

15. Occupational Safety and Health Administration. Occupational Safety and Health Standards 29 CFR 1910.151(c); 1998.

16. International Programme on Chemical Safety. Chemical Safety Information from Intergovernmental Organizations website. http://www.inchem.org/documents/pims/chemical/pimg022.htm\#SectionTitle:2.1 Main risk and target organs. Accessed March 2, 2015. 
17. Cheesbrough J, Green J, Gallimore C, Wright P, Brown D. Widespread environmental contamination with norwalk-like viruses (NLV) detected in a prolonged hotel outbreak of gastroenteritis. Epidemiol Infect. 2000;125:93-98. norovirus infection affecting an Austrian nursing home and a hospital. Wien Klin Wochenschr. 2005;117:802-808.

19. Booth CM. Vomiting Larry: A simulated vomiting system for assessing environmental contamination from projectile vomiting related to norovirus infection. $J$ Infect Prev. 2014;15:176-180.

20. Marx A, Shay DK, Noel JS, et al. An outbreak of acute gastroenteritis in a geriatric longterm-care facility: Combined application of epidemiological and molecular diagnostic methods. Infect Control Hosp Epidemiol. 1999;20:306-311.

21. Al-Wazzan B, Salmeen Y, Al-Amiri E, Abul A, Bouhaimed M, Al-Taiar A. Hand hygiene practices among nursing staff in public secondary care hospitals in Kuwait: selfreport and direct observation. Med Princ Pract. 2011;20:326-331. 